\section{Space-time coding for high-throughput interleave division multiplexing aided multi-source co-operation}

\section{R. Zhang and L. Hanzo}

Designed is an error-resilient, yet high-throughput interleave division multiplexing based space-time code suitable for multi-source co-operation. Its achievable rate, power efficiency and flexibility have been characterised.

Introduction: Co-operative communications allow us to assign the multiple input multiple output (MIMO) elements to geographically separated co-operating sources (CSs), which are no longer prone to correlated fading, leading to the concept of virtual MIMOs (VMIMO) [1]. Space-time code (STC) based VMIMO designs [2] are attractive for employment in co-operative communications, where the CSs' independently fading signals jointly constitute an STC codeword. Recently, an interleave division multiplexing space-time code (IDMSTC) was proposed [3], and its potential existence in co-operative communications was hypothesised. This open problem motivated us to design and investigate the proposed IDM-STC in the context of co-operative communications. Our design of IDM-STC was specifically contrived for multi-source co-operation (MSC) [4], where joint coding of multiple sources is applied. We improved the spectral efficiency of our scheme, as seen in Fig. 1, by appropriately adopting the multilayer concept [3], where we treat each CS as an IDM-STC layer. Furthermore, we also designed a realistic signalling scheme required for exchanging the information among the CSs and contrast the benefit of IDM-STC to that of the traditional G4 STC design [5]. The novel contribution of this Letter is that we design an error-resilient, yet high-throughput IDM-STC scheme suitable for MSC and characterise its achievable rate, power efficiency and flexibility.

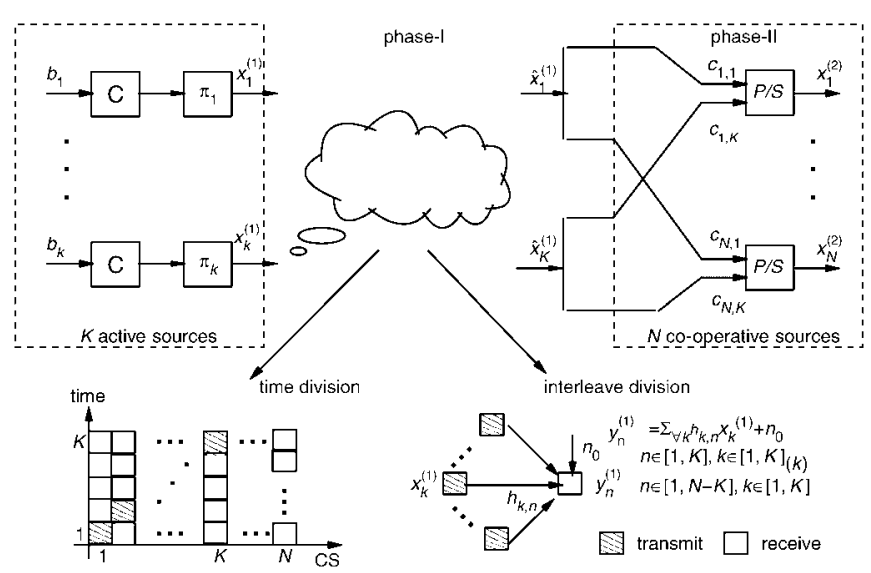

Fig. $1 \mathrm{~K}$-user co-operative IDM-STC UpLink transmitter

Multi-source co-operation: Consider a cluster of single-antenna sources co-operatively communicating with a destination employing a single receive antenna over independent identically distributed (IID) narrowband Rayleigh fading channels, resulting in a virtual multiple input single output (VMISO) system. In this VMISO cluster, we assume having a total of $N$ CSs, $K$ active sources (AS) and $(N-K)$ relaying sources (RS). We assume furthermore that each BPSK modulated AS employs a repetition code $\mathrm{C}$ of rate $R \leq 1 / N$ and a source-specific interleaver $\pi_{k}$.

Phase-I co-operation is defined as the information exchange of all $K$ ASs before transmitting the co-operatively combined IDM-STC signals. The $k$ th AS transmits a repetition coded and randomly interleaved bitstream $x_{k}^{(1)}=\pi_{k}\left[\mathrm{C}\left(b_{k}\right)\right], k \in[1, K]$. The superscript $(\cdot)^{(1)}$ denotes Phase-I co-operation. Two ways of Phase-I co-operation may be adopted. 1. Time division (TD), where a total of $K$ time-slots are assigned. At the $k$ th time-slot, the $n$th CS receives the signal transmitted from the $k$ th AS, $y_{k, n}^{(1)}=h_{k, n} x_{k}^{(1)}+n_{0}, k \in[1, K], n \in[1, N]_{(k)}$, where $n_{0}$ denotes the AWGN noise and $h_{k, n}$ is the flat-fading channel-coefficient between source $k$ and $n$. The subscript $[\cdot]_{(k)}$ denotes the exclusion of the $k$ th element. 2. By contrast in interleave division (ID) only one time-slot is required, because the simultaneously transmitted signals are differentiated by their interleavers. Among all the $N$ $\mathrm{CSs}$, the $n$th AS receives the signal $y_{n}^{(1)}=\sum_{\forall k} h_{k, n} x_{k}^{(1)}+n_{0}, k \in$ $[1, K]_{(k)}, n \in[1, K]$. The $n$th RS receives the signal $y_{n}^{(1)}=\sum_{\forall k} h_{k, n}$ $x_{k}^{(1)}+n_{0}, k \in[1, K], n \in[1, N-K]$.

Phase-II co-operation is defined as the joint transmission of a combined IDM-STC signal by the concerted action of all the $N$ CSs. Following Phase-I co-operation, each of the $N$ CSs detects all the $K$ ASs' bit-streams, resulting in $\hat{x}_{k}^{(1)}, k \in[1, K]$. When considering the $n$th of $N$ CSs, the joint IDM-STC codeword is constructed as follows: 1. The $n$th CS forms $K$ parallel streams $c_{n, k}^{(\mathrm{i})}=\hat{x}_{k}^{(1)}[N(i-1)+n], i \in$ $[1, M / N], k \in[1, K]$, where $M$ is the length of bit-stream $\hat{x}_{k}^{(1)}$; 2. These $K$ streams are parallel-to-serial $(\mathrm{P} / \mathrm{S})$ converted into $c_{n}$. Then the signal transmitted from the $n$th $\mathrm{CS}$ is $x_{n}^{(2)}(i)=\sum_{t=1}^{L_{n}} e^{j \theta_{n, l}}$ $c_{n}\left[L_{n}(i-1)+l\right], i \in\left[1, M K / N L_{n}\right]$, where $L_{n}$ is referred to as the number of layers contributed by the $n$th CS, while $\theta_{n, l}$ denotes the layer-specific phase rotation. In this Letter, we assume $L_{n}=L$ and $\theta_{n, l}$ $n \in[1, N]$.

An iterative receiver is employed at the destination for Phase-II co-operation, where either optimum but complex maximum-likelihood detection or suboptimum but simple interference cancellation may be employed [3]. The same receiver can also be employed for Phase-I co-operation using the ID scheme. Since the repetition code-rate obeys $R \leq 1 / N$, there is a sufficiently high degree of freedom for detecting $y_{n}^{(1)}$. However, this requires perfect channel knowledge at the co-operating receiver, which is a very demanding assumption, since estimating all the related channels imposes high complexity on the CSs. Thus, we could consider differentially encoded and non-coherently detected schemes, which can be used in the TD scheme of Phase-I co-operation at the cost of reducing the effective throughput by a factor of $K$, namely by the number of time-slots required.

Effective throughput: Consider having $N=K=4$ ASs in a cluster using a traditional $G_{4}$ type STBC generator matrix (GM) [5]:

$$
\mathbf{G}_{4}=\left[\begin{array}{cccccccc}
-x_{4}^{*} & -x_{3}^{*} & -x_{2}^{*} & x_{1}^{*} & -x_{4} & -x_{3} & -x_{2} & x_{1} \\
-x_{3}^{*} & x_{4}^{*} & x_{1}^{*} & x_{2}^{*} & -x_{3} & x_{4} & x_{1} & x_{2} \\
x_{2}^{*} & x_{1}^{*} & -x_{4}^{*} & x_{3}^{*} & x_{2} & x_{1} & -x_{4} & x_{3} \\
x_{1}^{*} & -x_{2}^{*} & x_{3}^{*} & x_{4}^{*} & x_{1} & -x_{2} & x_{3} & x_{4}
\end{array}\right]
$$

The traditional STBC used in MSC operates as follows: 1. exchange the information of $x_{k}, k \in[1, K]$, which requires $K$ time-slots; 2 . joint transmission of $x_{k}, k \in[1, K]$ using all $N$ CSs according to the above matrices, where the $n$th CS transmits the $n$th row of the above GM. For the sake of achieving high throughput, each symbol $x_{k}$ can be modulated onto an $\mathcal{M}$-ary constellation. The effective throughput per CS excluding the overhead of the Phase-I co-operation can thus be defined as $\eta_{S T B C}=r_{S T B C} \times\left(\log _{2} \mathcal{M}\right) / K$, where $r_{S T B C}=1 / 2$ is the rate of the $G 4$ STBC. In the IDM-STC considered, the overhead imposed by the TD scheme in Phase-I co-operation is constituted by $K$ time-slots, which is equivalent to that of the traditional STBC. The effective throughput per CS, excluding the overhead of Phase-I co-operation may be expressed as $\eta_{I D M}=r_{I D M} \times L / K$, where $r_{I D M}=$ $N \times R$ denotes the rate of IDM-STC.

Benefits of IDM-STC: We assume error-free detection during Phase-I co-operation and perfect knowledge of the channel between the CSs and the distant receiver at the destination. Two propagation scenarios are investigated: namely, 1. fast fading: the channels exhibit a normalised Doppler frequency of $f_{d}=0.02$. 2); block fading: the channels remain constant over each block but change between different blocks. We normalised the total transmit power of all $N$ CSs to ensure that it was unity in both scenarios for fair comparison.

Benefit 1 - Efficiency. Let us compare IDM-STC having $R=1 / 8$ to the above $G_{4}$ STBC in MSC, where we have $K=N=4$. In Fig. 2, $K=$ $1 \mathrm{AS}$ assisted by $N=4 \mathrm{CSs}$ using IDM-STC was characterised, which served as our benchmarker. Fig. 3 shows the achievable bit error ratio (BER) performance of IDM-STC in the block-fading scenario. Although a reduced performance was observed for IDM-STC compared to that of the fast-fading scenario of Fig. 2 owing to the lack of time diversity, they are both superior to that of the $G_{4}$ STBC. It can be seen in both Figs. 2 and 3 that the maximum number of layers supported was $L=7$, which has the same throughput as a $G_{4}$ STBC scheme using a large and hence error-sensitive 128-QAM constellation, and yet IDMSTC requires a lower power than the lower-throughput 4 bits $/$ symbol $\mathrm{G}_{4}$ 
STBC aided 16-QAM scheme, as observed at BER $\leq 10^{-5}$. Thus a significant power gain as well as rate gain can be observed compared to the $G_{4}$ STBC both under fast-fading as well as block-fading conditions.

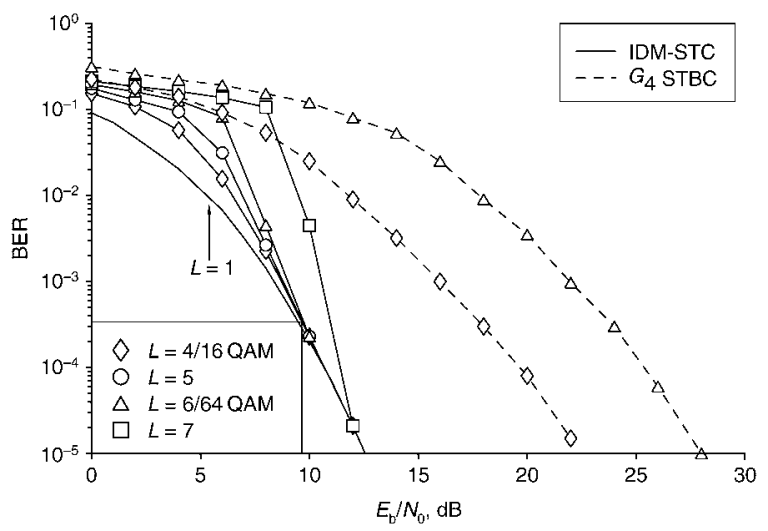

Fig. 2 Performance of $G_{4} S T B C$ and IDM-STC over fast fading in co-operative communications, where $L=4,5,6,7$ layers corresponding up to 7 bits/symbol transmission were supported

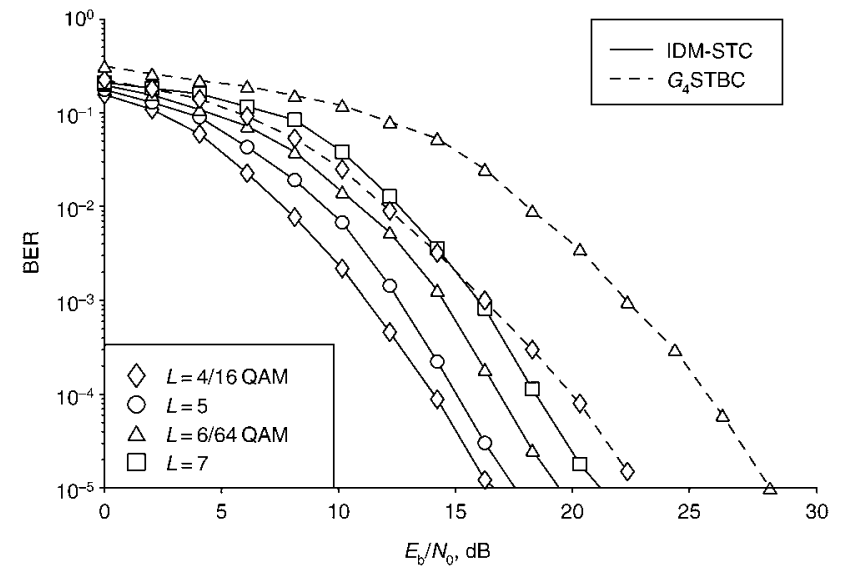

Fig. 3 Performance of $G_{4} S T B C$ and IDM-STC over block fading in co-operative communications, where $L=4,5,6,7$ layers corresponding up to 7 bits/symbol transmission were supported
Benefit 2 - Flexibility. The design flexibility of IDM-STC allows employment of an arbitrary number of CSs without designing different GMs when the traditional STBC code is employed. This implies that IDM-STC based MSC can be used in diverse co-operative scenarios. Suffice to say, however, that in most practical scenarios having a diversity order of more than five attains a near-AWGN BER performance and hence there is limited benefit in further increasing the diversity order, i.e. the number of CSs in particular, when considering the effective throughput reduction imposed by the Phase-I co-operation. This flexibility is beneficial in terms of forming a flexible cluster of CSs, allowing sources to freely join or disjoin the cluster of MSCs.

Conclusion: Compared to the traditional STBC based MSC design, our proposed IDM-STC design has a high rate, power efficiency and is flexible in terms of forming a cluster of CSs.

Acknowledgments: The financial support of the Mobile VCE and that of the EPSRC is gratefully acknowledged.

(C) The Institution of Engineering and Technology 2008 20 October 2007

Electronics Letters online no: 20083657 doi: 10.1049/el:20083657

R. Zhang and L. Hanzo (School of ECS, University of Southampton, SO17 1BJ, United Kingdom)

E-mail: lh@ecs.soton.ac.uk

\section{References}

1 Sendonaris, A., Erkip, E., and Aazhang, B.: 'User cooperation diversity. Two Parts', IEEE Trans. Commun., 2003, 51, pp. 1927-1948

2 Stefanov, A., and Erkip, E.: 'Cooperative space-time coding for wireless networks', IEEE Trans. Commun., 2005, 53, pp. 1804-1809

$3 \mathrm{Wu}, \mathrm{K}$., and Ping, L.: 'Multilayer turbo space-time codes', IEEE Trans. Commun., 2005, 9, pp. 55-57

4 Shalvi, O.: 'Multiple source cooperation diversity', IEEE Commun. Lett., 2004,8 , pp. $712-714$

5 Hanzo, L., Liew, T., and Yeap, B.: 'Turbo coding, turbo equalisation and space-time coding for transmission over fading channels' (Wiley-IEEE Press, 2002) 